\title{
A source for microhydrated biomolecules
}

\author{
M. Förstel, ${ }^{1, \text { a) }}$ M. Neustetter, ${ }^{2}$ S. Denifl, ${ }^{2}$ F. Lelievre, ${ }^{3,1}$ and U. Hergenhahn ${ }^{1, \text { b) }}$ \\ 1) Max-Planck-Institut für Plasmaphysik, Wendelsteinstraße 1, 17491 Greifswald, \\ Germany \\ ${ }^{2)}$ Institut für Ionenphysik und Angewandte Physik, Technikerstraße 25, \\ 6020 Innsbruck, Austria \\ 3) University Paris-Sud 11, Faculté des Science d'Orsay, 91405 Orsay, \\ France
}

(Dated: 16 June 2015)

We describe the construction of an apparatus for the production of a molecular jet of microhydrated biomolecules. Our design uses a water reservoir producing water vapour, which then passes through a separate reservoir containing a vapour of a sublimated biomolecule. The mixture coexpands into a molecular beam apparatus through a conical nozzle. Mass spectra showing water-adenin and water-uracil complexes are shown as typical examples. Suitable expansion conditions are reached without the use of an inert carrier gas.

Keywords: Nucleic-acid bases; water clusters; microhydration

\footnotetext{
a) Current address: University of Hawai'i at Manoa, 2545 McCarthy Mall, 96816 HI Honolulu, USA

b) Mailing address: IPP, c/o Helmholtz-Zentrum Berlin, Albert-Einstein-Str. 15, 12489 Berlin, Germany; E-Mail: uwe.hergenhahn@ipp.mpg.de
} 
The elementary molecules which make up living matter, such as the DNA bases and the amino acids, readily form complexes with one or several water molecules. Such 'microhydrated' biomolecules are important prototypes for experimental and computational approaches to biochemical function. Spectroscopic studies of their properties therefore are of interest, which as a prerequisite calls for methods to produce them in a form suitable for investigation. For mass spectrometry, electron spectroscopy etc., gas phase isolated complexes are required. In this note we describe the construction and operation of our source for neutral, gaseous organic molecules decorated with one or a few water molecules. Studies we aim at are charged particle spectroscopy (photoelectron spectroscopy, mass spectroscopy, electron-ion coincidence) after excitation by synchtrotron radiation. Our design is capable to produce a continuous stream of gas phase complexes of sufficient intensity for this purpose.

Earlier spectroscopic studies of microhydrated biomolecules used excitation and ionization by lasers in different combinations. In these experiments, biomolecules were sublimated into a stream of an inert carrier gas which was directed first through a water reservoir in order to moisten it. As examples for many such studies see ref.s 1, 2. Given the orders of magnitude lower values for average flux, and in particular peak photon flux, of synchrotron radiation compared to modern short-pulse laser systems, we do not believe this method would work for our intended experiments, although we are not aware of hard figures published for the density of microhydrated species in laser studies. Earlier works of larger relevance for our approach are by Kim et $a l .{ }^{3}$ and Belau et $a l .{ }^{4}$ Although different in detail, in both of these groups a vapour of biomolecules was produced by sublimation at the tip of a supersonic expansion nozzle, through which it streams together with water molecules and, optionally, a carrier gas. In ref. 4, fragments of the microhydrated complexes were detected by mass spectrometry after ionization in a single photon process using synchrotron radiation. Our set-up is inspired by this work.

For completeness we mention that mixed hydrated species cannot only formed by coexpansion, but also by attachment of molecules to pre-formed clusters after the expansion ('pick-up'). Pick-up of water molecules by molecular clusters ${ }^{5}$ as well as pick-up of vapourized atoms by water clusters ${ }^{6}$ have been used. Water complexes containing a single biomolecule have been produced such. ${ }^{7,8}$

The construction of our source is shown in Fig. 1. It basically consists (from right to left) of 


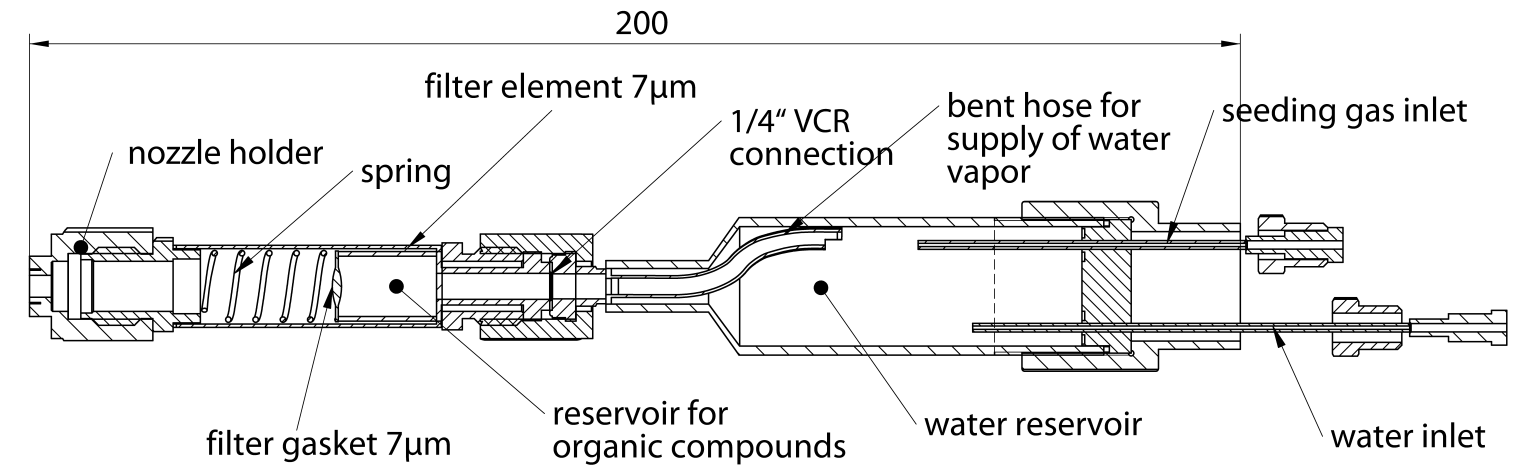

FIG. 1. Construction drawing of our source for hydrated biomolecules. Shown is a cut along the vertical plane containing the central axis. The source can be operated horizontally, producing a gas jet propagating to the left, or vertically pointing upward. See text for details.

a water reservoir, a reservoir for the dopant and a conical nozzle in which the clusters form. Within our apparatus, the source is placed on a three-axis manipulator inside the expansion chamber. The beam emitted by the nozzle is travelling through a skimmer (Beam Dynamics) into a second chamber where it is probed by synchrotron radiation. Further information on the vacuum chamber has been given. ${ }^{9}$ Data shown in this report were recorded with a commercial quadrupole mass spectrometer (QMS, Hiden), using electron impact to ionize the molecules in the gas jet. The source region of the QMS was placed at the approximate point of interaction with the synchrotron beam inside our chamber, and the axis of the QMS was aligned with the molecular beam.

Important characteristics of our design are the bent tube connecting the water reservoir to the dopant, which is a powder at room temperature, as well as the ability to separately control the temperature of the two reservoirs and the nozzle. The water reservoir consists of a custom made steel tube sealed at the end with a lid screwed onto the main cylinder. Into the lid two VCR connectors (Cajon) for 1/16" outer diameter steel tubes are welded, attached at different height. These connect to vacuum feedthroughs, closed off with valves. The lower tube (in the orientation of the drawing) is used to fill the reservoir, while the upper one can be used to pump out excess water vapour or to additionally feed in a seeding gas. (Seeding gas was not used in the experiments shown here.) It is thus possible to fill in water without breaking the vacuum. Usually, the under-pressure due to the vacuum of the 
expansion chamber is sufficient to transport water into the reservoir once the valve in the feedline is opened. When it is heated, the resulting water vapour will expand through the bent tube into the biomolecule reservoir. This design separates the water vapour from the liquid and prevents that the nozzle accidentally is reached by water droplets, which would result in a massive overpressure in the expansion chamber. Neat water clusters (dopant reservoir empty) form at conditions comparable to earlier experiments (e.g. 10), and show the expected series of singly protonated fragments. ${ }^{11}$

The nozzle itself has a conical cross section with a half opening angle of $15^{\circ}$ and a thickness of $1.1 \mathrm{~mm}$, and has been produced from copper (Günther Frey Präzisionsmechanik). ${ }^{12}$ The diameter at the throat of the expansion is $80 \mu \mathrm{m}$. It is screwed onto a 4-VCR nut connected to the filter holder.

A lot of small molecules of biological relevance have the form of a powder when they are isolated at room temperature. Typical sublimation temperatures to reach a vapour pressure in the some tens of mbar range are on the order of $540 \mathrm{~K} \cdot{ }^{13,14}$ Vapour phase experiments using several amino acids and DNA bases at significantly higher temperatures did not find signs of thermal decomposition. ${ }^{15,16}$ Our reservoir for these molecules consists of a commercial filter element for gas streams, equipped with a metal sintered cartridge with a pore size of $7 \mu \mathrm{m}$ (Swagelok F-type). Towards the open side of the cartridge, a filter gasket with a similar pore size is inserted. Refilling the biomolecule reservoir requires to take the source out of the expansion chamber and to open the reservoir at the nozzle side.

The two reservoirs and the nozzle are heated each by a mineral insulated resistive heater (Thermocoax). For the water reservoir, typically having the lowest temperature, we have also used simple resistive heating wire. Temperatures are controlled by three K-type thermocouples. Manual fine control of the heating power is needed to keep the temperatures constant within a few K. This could be avoided by implementing a feedback loop for the heating power, which was not attempted here.

A photograph of the assembled source is shown in Fig. 2.

Some notes on the operation of the source follow. To prevent clogging it is important to keep the temperature of the nozzle higher than the biomolecule reservoir. Thus, when turning on the source, first the nozzle should be heated up to about $323 \mathrm{~K}$ before turning on the heating of the reservoir. Taking into account that the biomolecule will decompose or burn at elevated temperatures, the maximum power to the heating wire should not be 


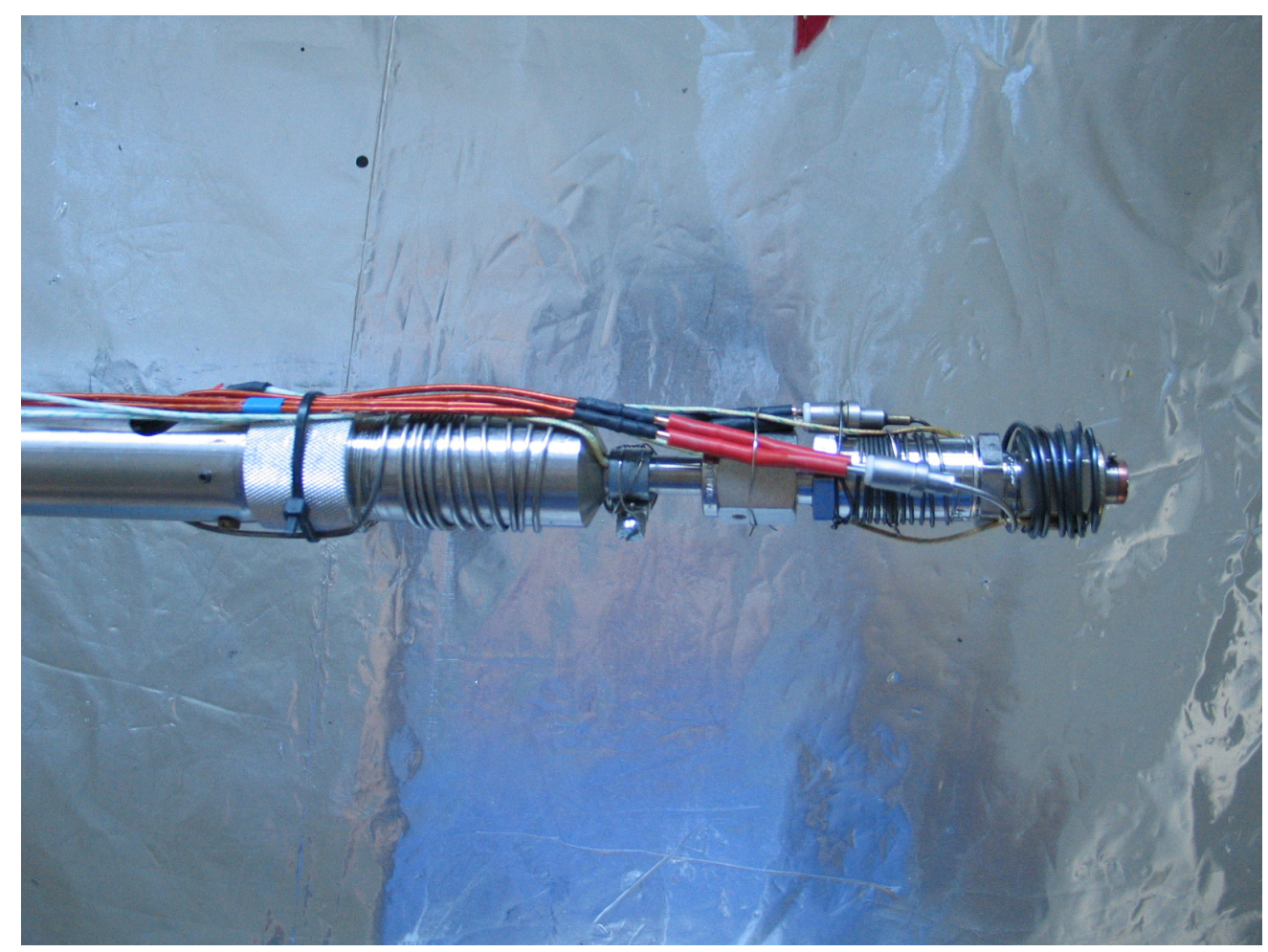

FIG. 2. Photograph of the source shown in Fig. 1.

higher than $9 \mathrm{~W}$. The nozzle itself can be heated up with a maximum power of $11 \mathrm{~W}$. The operating temperatures are dependent on the compound one is interested in. For example, we found that adenine needs at least a reservoir temperature of $518 \mathrm{~K}$ to achieve efficient formation of clusters (see Fig. 3).

As mentioned before, it is important that the nozzle is hotter than the biomolecule reservoir. Due to thermal conduction the temperature difference in our case is limited to $20 \mathrm{~K}$. However, we mainly worked with differences between 10 and $15 \mathrm{~K}$. Once the operating temperatures are reached they can be kept constant with a power of 6 to $8 \mathrm{~W}$. The maximum power we applied to the water container was $12 \mathrm{~W}$. During our tests we never heated it up higher than $423 \mathrm{~K}$, providing an expansion pressure of about 4.5 bar. $^{18}$ The minimum temperature of the water container is dependent on the temperature of the biomolecule reservoir (typically heated higher than the former). This is due to thermal conduction. Nevertheless, during the measurements we were able to keep differences of about 140K without problems. For a supersonic expansion source the stability of the expansion pressure is important to have stable conditions. Thus, due to the consumption of water from the container during operation, it is necessary to adjust the power at the water container 


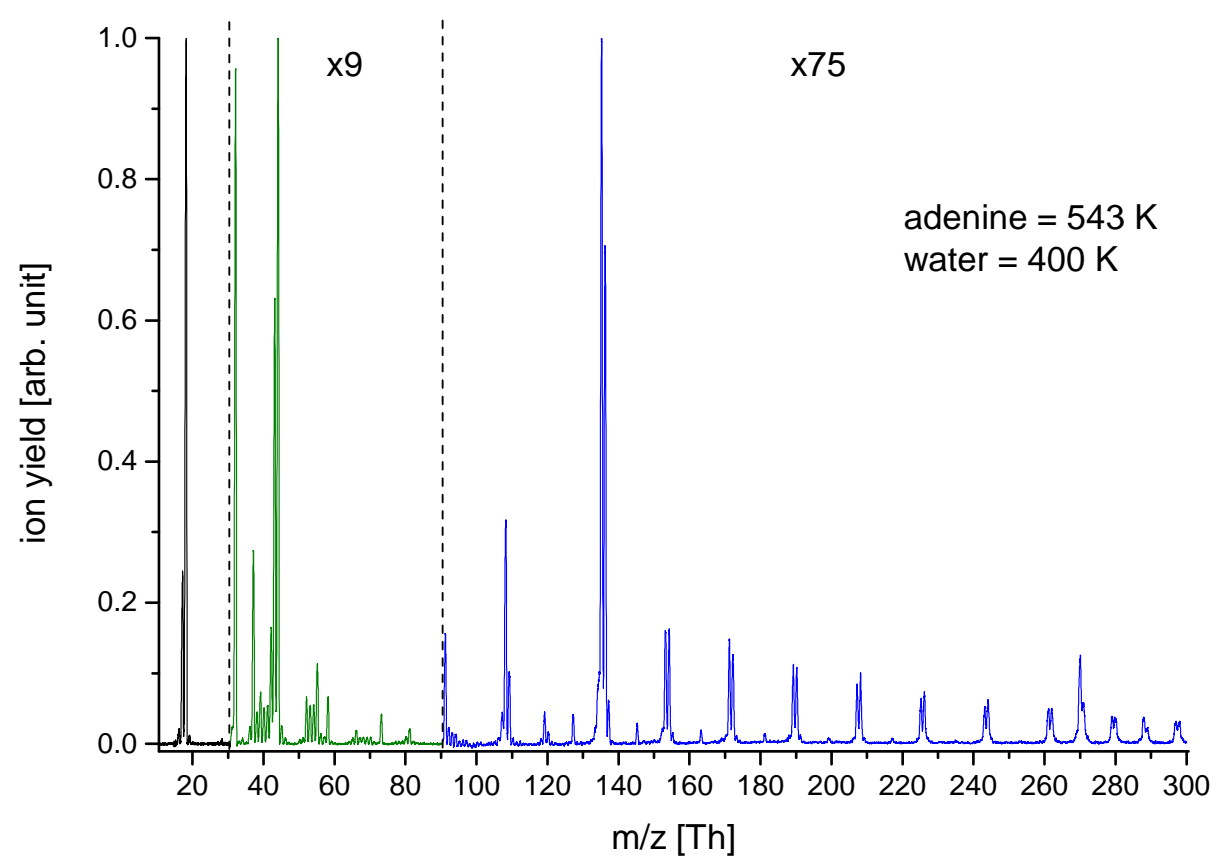

FIG. 3. Spectrum of an adenine-water mixture in the full mass range of 10 to $300 \mathrm{amu}$ obtained at an electron energy of $70 \mathrm{eV}$. The graph is assembled of measurements with three different sensitivities of the mass spectromer as indicated by the dashed lines. Each of the three regions has been normalized on its most intense peak. In the present spectrum we can see the bare adenine monomer at 135 amu as well as the dimer. Both species we found solvated by water molecules and both series we observed protonated as well as non-protonated. At smaller intensity, also a series of singly protonated water clusters without presence of an adenine molecule is detected $(91,109,117$, ... amu). The fragment at $108 \mathrm{amu}$ is known from the mass spectrum of pure adenine. ${ }^{17}$ For future studies the mass range above the monomer, where the hydrated species is seen, will be most important.

frequently. Refilling the water leads to a fast decrease of the container temperature of about 30 to $40 \mathrm{~K}$ (depending on the amount and temperature of the refilled water). With a full water container we were able to measure at least about $3.5 \mathrm{~h}$. To shorten the dead time, water can be injected into the container at elevated temperatures. In measurements with uracil extending over more than 48 h, the biomolecule reservoir had to be refilled after 12 h, approximately.

To test and characterize our source, we used a commercial QMS with a maximum mass 
range of $300 \mathrm{amu}$. The ions were produced via electron impact ionization, with an ionization energy of $70 \mathrm{eV}$. Cluster source and ion source chamber were separated by a skimmer with a diameter of $1 \mathrm{~mm}$, which allowed differential pumping. The distance between orifice and skimmer was set to about $10 \mathrm{~mm}$. With this setup, test measurements were made with uracil, thymine and adenine.

In the following, we briefly discuss a cluster distribution of uracil, shown in Fig 4, in the mass range of 100 to 300 amu. For this spectrum the biomolecule was heated up to a temperature of about $513 \mathrm{~K}$ whereas the nozzle temperature was kept at about $528 \mathrm{~K}$ and the water container temperature at about $403 \mathrm{~K}$, respectively. The pressure in the expansion chamber and in the QMS region was $2 \times 10^{-4}$ mbar and $7 \times 10^{-7} \mathrm{mbar}$, respectively.

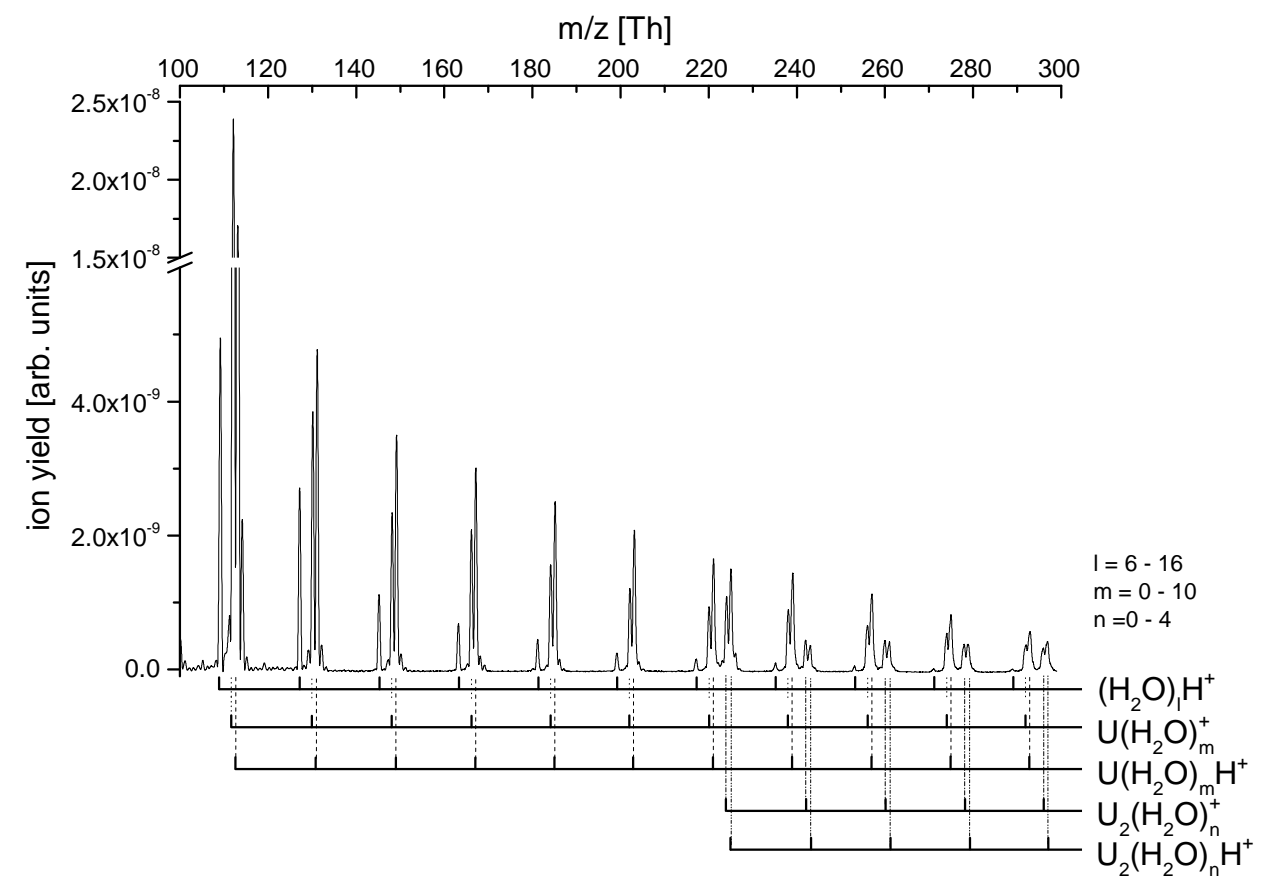

FIG. 4. Representative mass spectrum of a uracil-water measurement in the range of 100 to 300 amu (uracil monomer: $112 \mathrm{amu}$ ). The graph shows a combination of two different spectra which were both recorded at an electron energy of $70 \mathrm{eV}$, with a different sensitivity of the QMS. Five different cluster series are distinguishable. Those are the bare protonated water clusters and uracilwater mixtures, for uracil monomer and dimer, both kinds protonated as well as non-protonated.

In Fig. 4, five different cluster series containing pure water clusters and uracil-water 
mixtures can be distinguished. In case of the neat water-cluster series we can only see the protonated species $\left(\left(\mathrm{H}_{2} \mathrm{O}\right)_{1} \mathrm{H}^{+}\right)$which is well-known. ${ }^{11}$ Water clusters with $l=6$ up to 16 molecules are visible in Fig. 4. Another series is obtained for the uracil monomer $\left(\mathrm{U}\left(\mathrm{H}_{2} \mathrm{O}\right)_{\mathrm{m}}^{+}\right)$ solvated with up to $m=10$ water molecules. Above the mass of the uracil dimer (224 amu) also hydrated uracil dimers $\left(\mathrm{U}_{2}\left(\mathrm{H}_{2} \mathrm{O}\right)_{\mathrm{n}}^{+}\right)$are visible. For uracil monomers as well as dimers, protonated and non-protonated species can be observed. The intensity of protonated monomers $\left(\mathrm{U}\left(\mathrm{H}_{2} \mathrm{O}\right)_{\mathrm{m}} \mathrm{H}^{+}\right)$is always higher than the intensity of the non-protonated parent ion, presumably $\left(\mathrm{U}\left(\mathrm{H}_{2} \mathrm{O}\right)_{\mathrm{m}+1} \mathrm{H}^{+}\right)$. The presence of the protonated species indicates that the hydrated clusters undergo efficient fragmentation during the ionization process. We note that the situation is slightly different in the case of the dimer $\left(\mathrm{U}_{2}\left(\mathrm{H}_{2} \mathrm{O}\right)_{n} \mathrm{H}^{+}\right)$, where the ion yields of non-protonated and protonated fragments at least for $n=1$ to 3 are close to each other. It seems that in this case the presence of the second uracil in the cluster prevents fragmentation.

We have used the source described here for a study of the fragmentation of hydrated uracil complexes by synchrotron radiation in the vacuum ultraviolet range. The cluster size distribution, as inferred from the ratio of the $m=0$ to the $m=1$ fragment, varied by less than $30 \%$ within a period of $16 \mathrm{~h}$. Over the course of these experiments the source was operated for more than $72 \mathrm{~h}$ continuously, interrupted only for refilling the reservoirs. More detailed results will be reported elsewhere. ${ }^{19}$

Summarizing, we have constructed and operated a source that produces a jet of hydrated biomolecules in vacuum. Behind a skimmer, the density is sufficient for probing with comparatively thin radiation sources, such as synchrotron radiation from a bending magnet. Our set-up does not require the use of an inert carrier gas.

\section{ACKNOWLEDGMENTS}

The authors gratefully acknowledge funding by the DFG research unit FOR 1789 and by the FWF (I1015). 


\section{REFERENCES}

${ }^{1}$ A. Weichert, C. Riehn, H.-D. Barth, G. Lembach, M. Zimmermann, B. Brutschy, and D. Podenas, Rev. Sci. Instrum. 72, 2697 (2001).

${ }^{2}$ Y. Nosenko, M. Kunitski, C. Riehn, P. H. P. Harbach, A. Dreuw, and B. Brutschy, Phys. Chem. Chem. Phys. 12, 863 (2010).

${ }^{3}$ S. K. Kim, W. Lee, and D. R. Herschbach, J. Phys. Chem. 100, 7933 (1996).

${ }^{4}$ L. Belau, K. R. Wilson, S. R. Leone, and M. Ahmed, J. Phys. Chem. A 111, 7562 (2007).

${ }^{5}$ L. Poth, Z. Shi, Q. Zhong, and A. W. Castleman Jr., Int. J. Mass Spectrom. 154, 35 (1996).

${ }^{6}$ C. Bobbert and C. P. Schulz, Eur. Phys. J. D 16, 95 (2001).

${ }^{7}$ R. Moro, R. Rabinovitch, and V. V. Kresin, Rev. Sci. Instrum. 76, 056104 (2005).

${ }^{8}$ R. Moro, R. Rabinovitch, and V. V. Kresin, J. Chem. Phys. 123, 074301 (2005).

${ }^{9} \mathrm{M}$. Mucke, Employing electron-electron coincidence techniques to investigate the autoionisation of clusters, Ph.D. thesis, Technical University Berlin (2011), http://opus.kobv.de/tuberlin/volltexte/2011/3073/.

${ }^{10}$ S. Barth, M. Oncak, V. Ulrich, M. Mucke, T. Lischke, P. Slavicek, and U. Hergenhahn, J. Phys. Chem. A 113, 13519 (2009).

${ }^{11}$ O. Echt, D. Kreisle, M. Knapp, and E. Recknagel, Chem. Phys. Lett. 108, 401 (1984).

${ }^{12}$ S. Marburger, Experimentelle Untersuchungen zum Interatomaren Coulomb Zerfall an Neon Clustern: Nachweis eines ultraschnellen nichtlokalen Zerfallskanals, Ph.D. thesis, Technical University Berlin (2004), http://opus.kobv.de/tuberlin/volltexte/2004/756.

${ }^{13}$ D. Holtkamp, W. Lange, M. Jirikowsky, and A. Benninghoven, Appl. Surf. Sci. 17 (1984).

${ }^{14}$ A. de Barros, A. Medina, F. Zappa, J. Pereira, E. Bessa, M. Martins, L. Coelho, W. Wolff, and N. de Castro Faria, Nucl. Instrum. Meth. A 560, 219 (2006).

${ }^{15}$ P. Colarusso, K. Zhang, B. Guo, and P. F. Bernath, Chemical Physics Letters 269, 39 (1997).

${ }^{16}$ I. Powis, E. E. Rennie, U. Hergenhahn, O. Kugeler, and R. Bussy-Socrate, J. Phys. Chem. A 107, 25 (2003).

${ }^{17}$ d. NIST Mass Spec Data Center, S.E. Stein, "'mass spectra' in nist chemistry webbook, nist standard ref. database no. 69," (National Institute of Standards and Technology, Gaithersburg MD, 20899) http://webbook.nist.gov, (retrieved May 8, 2015). 
${ }^{18}$ W. Wagner and A. Pruss, J. Phys. Chem. Reference Data 22, 783 (1993).

${ }^{19}$ N. Wechselberger, M. Neustetter, U. Hergenhahn, T. Jahnke, and et al, To be published. 


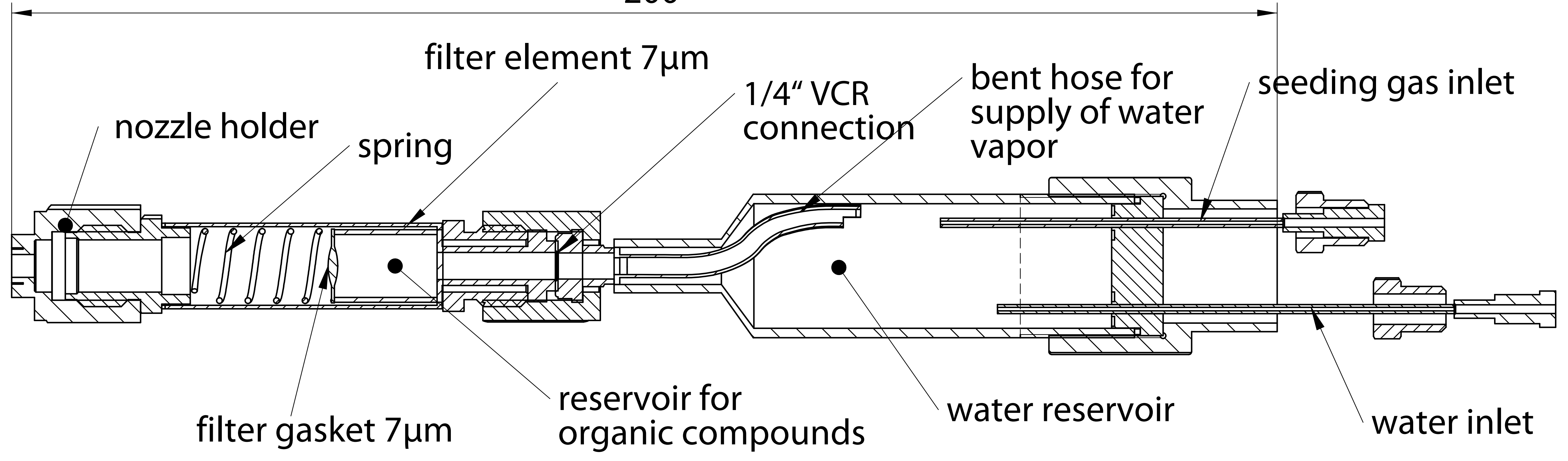

